\title{
Bien vieillir
}

\section{La voie de signalisation insuline-FOXO et la longévité}

> Le vieillissement est un processus complexe qui s'accompagne de maladies liées à l'âge comme la maladie d'Alzheimer. Le vieillissement est contrôlé par une combinaison de facteurs génétiques et environnementaux. Parmi les voies cellulaires qui régulent le vieillissement, la voie de signalisation insuline-FOXO joue un rôle central car elle contrôle la durée de vie dans de multiples espèces animales comme le ver, la mouche et la souris. Chez l'homme, la longévité exceptionnelle - le fait d'être centenaire - est également associée à des variations génétiques de la voie insuline-FOXO. Des études récentes ont révélé que les facteurs de transcription FOXO jouent un rôle clé dans le renouvellement des cellules souches adultes et embryonnaires, ce qui pourrait contribuer à la régénération des tissus adultes. Comprendre les mécanismes du vieillissement pourrait aider à mieux prévenir et traiter les maladies liées à l'âge. <

Le processus de vieillissement s'accompagne de l'apparition d'une série de pathologies liées à l'âge, incluant la maladie d'Alzheimer, le cancer et les maladies cardio-vasculaires. L'âge est notamment le facteur de risque principal pour la maladie d'Alzheimer. À partir de 85 ans, plus de $40 \%$ de la population présente des signes d'altération de la mémoire associés à la maladie d'Alzheimer ou à d'autres formes de démences séniles. Le vieillissement s'accompagne également d'un déclin des fonctions physiologiques, même chez l'individu sain. Ainsi, chez l'homme, la mémoire à court terme et la vitesse d'intégration de l'information déclinent avec l'âge, et ce même en l'absence de maladies neurodégénératives. Une question fondamentale se pose donc: le vieillissement peut-il être régulé ? Comprendre les facteurs qui contrôlent le vieillissement pourrait permettre d'appréhender les maladies liées à l'âge sous un autre angle et de soigner non pas les symptômes de ces maladies mais leur cause principale : le vieillissement.

\section{Anne Brunet}

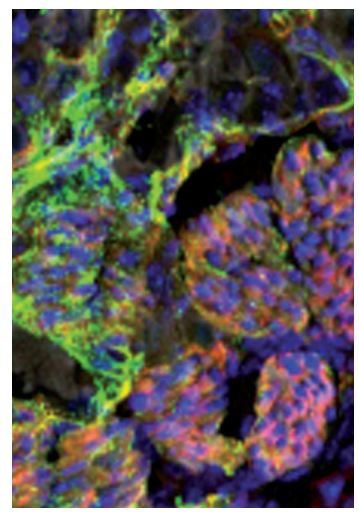

Département de génétique, Stanford University, 300 Pasteur drive, 94305 Stanford, CA, États-Unis abrunetl@stanford.edu

Pendant longtemps, le vieillissement n'a pas été considéré comme un processus biologique pouvant être régulé. Il était communément admis que le vieillissement était inévitable et dû à une usure généralisée de l'organisme. Mais cette vision quelque peu fataliste du vieillissement a été remise en question par les études génétiques dans de nombreux organismes qui montrent que le vieillissement peut être contrôlé par une combinaison de facteurs génétiques et environnementaux. Des études sur la longévité des jumeaux séparés à la naissance (génétiquement identiques mais vivant dans deux environnements différents) ont indiqué que la part génétique dans la régulation du vieillissement était de $30 \%$, alors que la part environnementale était de $70 \%$. Les toxines, les radiations, le stress et la pollution sont parmi les nombreux facteurs qui influencent le vieillissement de façon négative. À l'inverse, la restriction calorique est un facteur environnemental qui non seulement accrôit la durée de vie, mais aussi réduit l'incidence des pathologies liées à l'âge.

\section{Le récepteur de l’insuline : un facteur clé du vieillissement}

Une avancée fondamentale dans l'étude du vieillissement provient d'une découverte majeure par l'équipe de Cynthia Kenyon, à l'université de Californie à San Francisco. Elle a montré que des vers C. elegans ayant une mutation dans le récepteur de l'insuline pouvaient vivre deux à trois fois plus longtemps que des vers ne possédant pas cette mutation [1] (Figure 1). Non seulement ces vers mutants pour le récepteur de l'insuline vivent plus longtemps que les vers non mutés, mais ils vieillissent moins vite. C'est donc la période de jeunesse qui est augmentée chez ces vers, et non la période de déclin. Cette découverte est importante car elle montre que la mutation d'un seul gène peut conduire à un allongement de la longévité, en particulier de la période juvénile. 

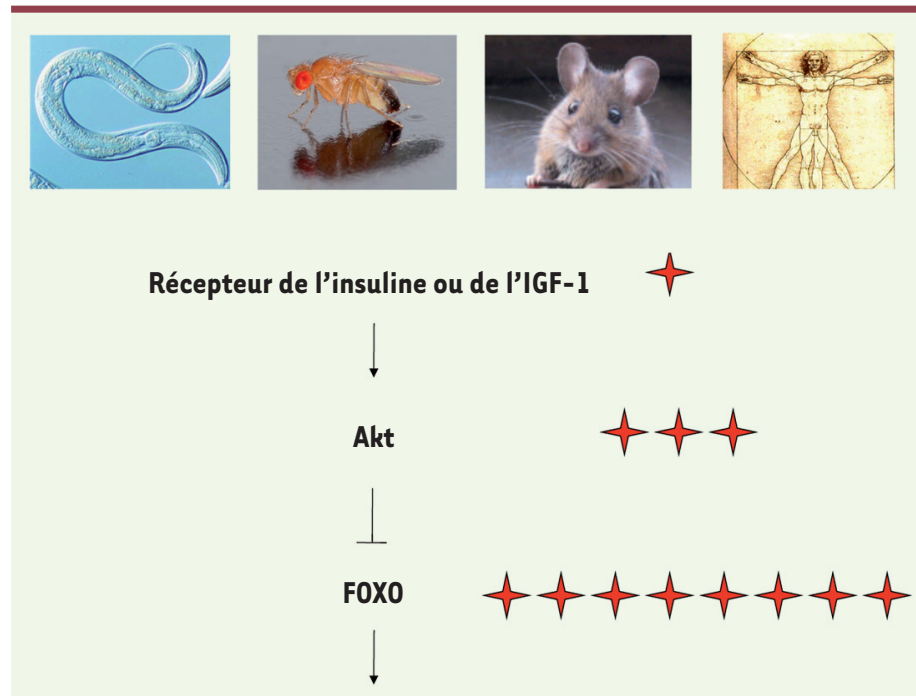

Régulation de la longévité

Figure 1. La voie de signalisation insuline-FOXO joue un rôle majeur dans la régulation de la longévité chez le ver, la mouche, la souris et l'homme. Les étoiles rouges indiquent les cohortes de centenaires qui présentent des variations génétiques dans les gènes cités (adapté de [2]).

De façon remarquable, l'effet d'une mutation du récepteur de l'insuline sur le prolongement de la durée de vie ne se limite pas au ver C. elegans [2]. Des études chez la mouche Drosophila melanogaster et chez la souris ont montré que des déficiences du récepteur de l'insuline, ou d'un récepteur apparenté, le récepteur de l'insulin-like growth factor 1 (IGF-1), prolongent également la durée de vie de ces organismes [3-6] (Figure 1). La mutation du récepteur de I'IGF-1 ralentit également l'apparition de la maladie d'Alzheimer dans un modèle murin de cette pathologie [7]. Chez l'homme, il est intéressant de noter que des mutations du récepteur de I'IGF-1 sont associées à des formes extrêmes de longévité chez les individus centenaires [8, 39] (Figure 1). Il semble donc que des mutations des récepteurs de l'insuline et de I'IGF-l s'accompagnent, chez le ver comme chez l'homme, d'un allongement de la durée de la vie.

\section{Les facteurs de transcription FOXO sont les cibles préférentielles de la voie de signalisation de l'insuline et de l'IGF-1}

Comment l'information reçue par ces récepteurs de l'insuline et de I'IGF-l est-elle relayée? Ces récepteurs sont activés par leur ligand (I'insuline ou I'IGF-1), ce qui induit l'activation d'une cascade de signalisation intracellulaire impliquant la phosphoinositide-3 kinase (PI3K), ainsi que d'autres protéine kinases activées en cascade: PDKl (3-phosphoinositide-dependent protein kinase-1), puis Akt (protéine kinase B) et SGK (serum and glucocorticoid-induced protein kinase) (Figure 2). Un élément majeur pour la transmission de ce signal dans le noyau de la cellule est la sous-famille de facteurs de transcription Forkhead appelée FOXO (pour forkhead box 0) [9, 40$]$.
En effet, Akt et SGK phosphorylent directement les facteurs de transcription FOXO sur trois résidus très conservés, depuis le ver jusqu'à I'homme [10-13]. La phosphorylation de FOXO par Akt et SGK se traduit par la séquestration de ces facteurs dans le cytoplasme via l'interaction avec la protéine chaperonne 14.3.3 (Figure 2) [10, 13]. De cette manière, la voie de l'insuline inhibe l'activité transcriptionnelle des facteurs de transcription FOXO.

En revanche, quand les niveaux d'insuline ou d'IGF-l sont bas, leurs récepteurs sont moins activés et la voie de signalisation de l'insuline est moins active. Dans ce contexte, les facteurs de transcription FOXO ne sont plus phosphorylés, ce qui induit leur translocation active dans le noyau de la cellule. Ils agissent à ce niveau principalement comme des activateurs transcriptionnels de gènes impliqués dans la détoxification des espèces réactives de l'oxygène, la réparation des dommages à I'ADN, l'apoptose et l'arrêt du cycle cellulaire [10, 13-17, 42] (Figure 2). Les facteurs de transcription FOXO induisent donc un «programme de réponse au stress » dans les cellules des mammifères, mais aussi chez le ver [18]. Ce programme de réponse au stress est important pour la capacité des FOXO à promouvoir la longévité, du moins chez le ver où cela a été testé [18].

\section{FOXO : un rhéostat cellulaire?}

Les facteurs de transcription FOXO intègrent une grande variété de signaux extracellulaires, en plus de l'insuline et de l'IGF-1 [19]. L'activité de ces facteurs est en effet modulée par des voies de signalisation déclenchées en réponse au stress oxydant ou nutritionnel ; c'est le cas notamment lors d'une carence énergétique, comme la restriction calorique, qui active une voie faisant intervenir l'AMP-activated protein kinase (AMPK) [20, 21]. Ces voies régulent FOXO par le biais de modifications post-traductionnelles incluant l'acétylation, l'ubiquitination et la phosphorylation d'une variété de résidus [20, 22-24]. L'impact de ces modifications sur la fonction de FOXO est encore mal compris, mais il semble que ces modifications puissent, dans certains cas, diriger le recrutement de ces facteurs de transcription sur les promoteurs de groupes spécifiques de gènes cibles. Enfin, les facteurs de transcription FOXO peuvent être également régulés de façon transcriptionnelle. Par exemple, le stress cellulaire augmente l'expression de I'ARN messager de FOX03, une des isoformes de FOXO chez les mammifères, et ce de manière dépendante du suppresseur de tumeur p53 [25]. Les facteurs de transcription FOXO jouent donc le rôle de rhéostat 


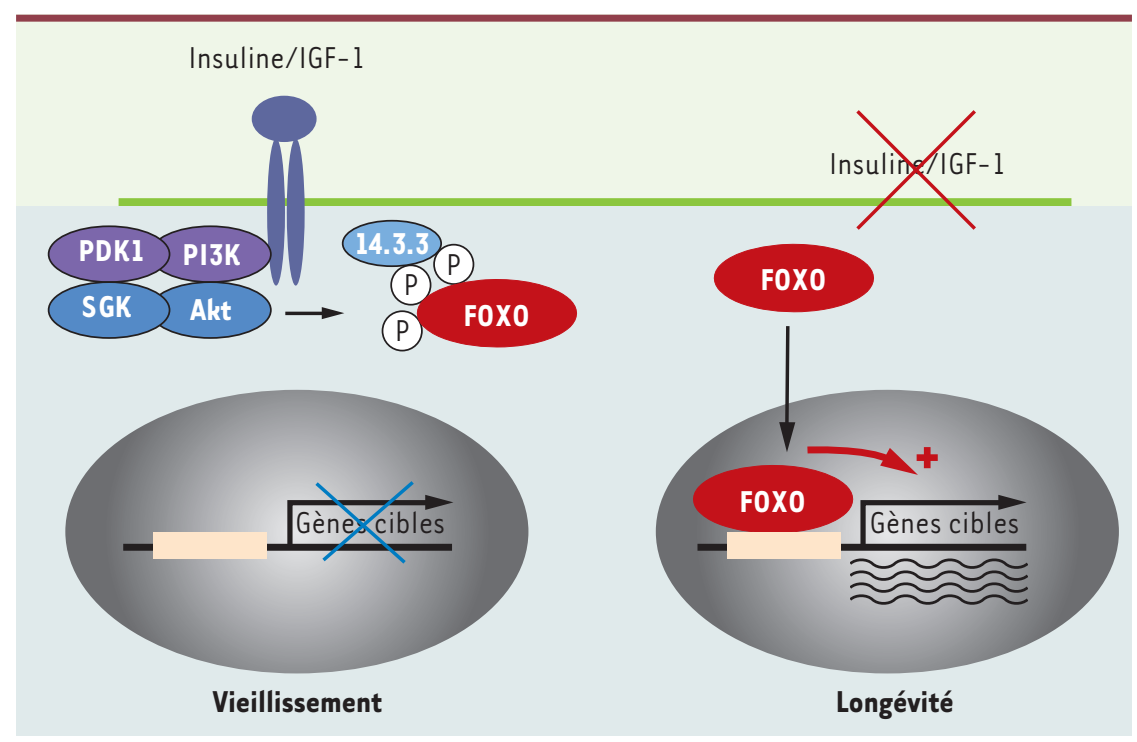

Figure 2. Régulation des facteurs de transcription FOXO par la voie insuline/IGF-1. Les facteurs de transcription FOXO sont séquestrés dans le cytoplasme quand les niveaux d'insuline et d'IGF-1 sont élevés, ce qui les empêche d'agir. En revanche, quand les niveaux d'insuline et d'IGFl sont bas, les facteurs de transcription FOXO sont transportés dans le noyau des cellules où ils induisent l'expression de gènes impliqués notamment dans la détoxification des espèces réactives de l'oxygène et la réparation des dommages à l'ADN, ce qui accroît la durée de vie.

cellulaire pour l'insuline et l'IGF-1, ainsi que pour le stress oxydant et nutritionnel.

\section{FOXO et la longévité extrême chez l'homme}

Chez le ver et la mouche, l'expression de FOXO suffit dans certaines circonstances à prolonger la durée de vie de ces organismes [26-28], ce qui définit FOXO comme « un gène de longévité ». Qu'en est-il chez l'homme? De façon très intéressante, des variants polymorphiques du gène codant pour le facteur de transcription F0X03 sont associés à la longévité exceptionnelle - le fait d'être centenaire - dans au moins huit cohortes indépendantes de centenaires, que ce soit en Europe, en Amérique ou en Asie [29-33] (Figure 1). II est frappant de constater que, de manière générale, ces centenaires conservent leur fonctions cognitives et échappent à la plupart des pathologies traditionnellement liées à l'âge, comme la maladie d'Alzheimer ou le cancer [29, 31 . Le polymorphisme de FOX03 responsable de cette longévité exceptionnelle n'est pas encore connu (pour l'instant, seuls des polymorphismes associés ont été décrits et ils sont présents dans les introns du gène FOX03). Toutefois, des études préliminaires suggèrent que les polymorphismes du gène FOXO3 responsables de l'allongement de la durée de vie n'affectent pas la séquence codante de la protéine F0X03. On peut donc faire l'hypothèse que les polymorphismes de FOXO3 liés à la longévité exceptionnelle pourraient influencer les niveaux d'expression du gène FOXO3. En accord avec cette hypothèse, les introns du gène $\mathrm{FOXO3}$ contiennent de nombreux sites de liaisons pour d'autres facteurs de transcription, comme $p 53$, connus comme régulateurs de l'expression du gène FOXO3 [25]. II est possible que ces sites soient directement affectés par un polymorphisme. Peut-être les centenaires ont-ils plus de F0X03 disponible dans leurs cellules parce qu'un facteur de transcription se lie mieux dans les régions régulatrices de ce gène ? De même, une plus grande quantité de $\mathrm{FOX03}$ pourrait être bénéfique chez l'homme, comme chez le ver ou la mouche, pour résister au stress oxydant.

\section{FOXO, les cellules souches et la longévité}

Les cellules souches offrent un paradigme intéressant pour la longévité et les facteurs de transcription FOXO. Ces cellules souches, qu'elles soient embryonnaires ou adultes, possèdent des capacités plastiques et régénératives exceptionnelles, à la différence des cellules différenciées. Cette caractéristique rend les cellules souches attractives pour identifier les processus de rajeunissement à l'œuvre dans ces cellules. L'individu adulte possède des réservoirs de cellules souches dans quasiment tous ses tissus, et ces cellules sont importantes pour la régénération de ces tissus (Figure 3 ). Par exemple, dans le cerveau des mammifères, il existe des réservoirs de cellules souches neurales qui sont importants pour la mémoire olfactive et spatiale. Les cellules souches neurales peuvent produire de nouveaux neurones chez l'adulte. Mais ces cellules perdent cette capacité avec l'âge (Figure 3). C'est également le cas des cellules souches hématopoïétiques qui perdent leur capacité de produire de nouvelles cellules sanguines au cours du vieillissement. Quel est le rôle des gènes de longévité dans les cellules souches ? Des études récentes ont montré que les facteurs de transcription FOXO ont un rôle clé dans la conservation de la fonction des cellules souches neurales et hématopoḯtiques [34-37, 41, 42] (Figure 3). En l'absence de FOXO, ces cellules souches adultes vieillissent prématurément, ce qui se traduit par des défauts de renouvellement et de différenciation. Plus récemment, un membre de la famille FOXO, F0X01, a été impliqué dans la capacité des cellules souches embryonnaires humaines à se différencier en multiples types cellulaires $[38,42](\rightarrow)$. II semble que les gènes régulés par FOXO $(\rightarrow)$ Voir la Nouvelle de P. Rimmelé et al., page 250 de ce numéro 


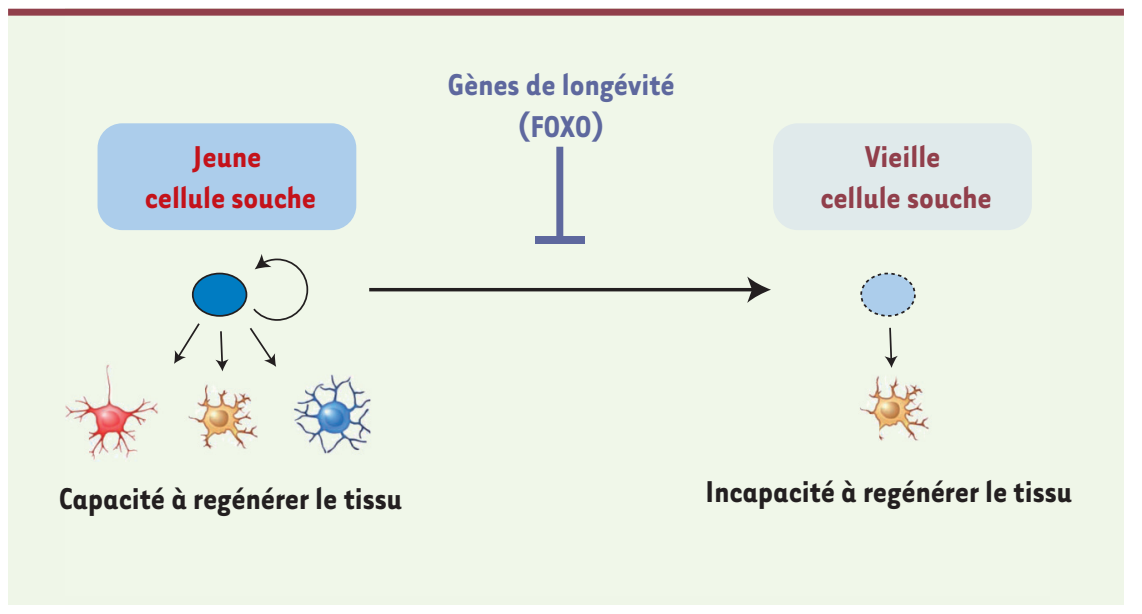

Figure 3. Rôle des facteurs de transcription FOXO dans le renouvellement des cellules souches. Les cellules souches de l'individu jeune participent au renouvellement des tissus en produisant des cellules différenciées. En revanche, chez l'individu âgé, ces cellules souches sont moins aptes à renouveler les tissus. Les facteurs de transcription FOXO assurent la pérennité des propriétés des cellules souches neuronales et hématopoiétiques, ce qui peut aider à préserver la qualité des tissus dans lesquelles ces cellules sont présentes (système nerveux et sanguin) au cours de la vie.

dans différentes cellules souches dépendent du type de cellule souche impliqué. II sera intéressant de comprendre le mode d'action des facteurs FOXO dans ces cellules: quels gènes cibles sont communs à toutes les cellules souches et quels gènes cibles sont spécifiques à certaines? Comprendre le réseau transcriptionnel enclenché par FOXO dans les cellules souches permettra d'identifier des cibles potentielles pour maintenir une réserve de ces cellules à fort potentiel régénératif de façon prolongée dans l'organisme. Il est également tentant de spéculer que le rôle de FOXO dans les cellules souches contribue à la longévité, ou tout au moins à la préservation de la qualité des tissus au cours du vieillissement.

\section{Conclusion et questions pour l'avenir}

La voie de signalisation de l'insuline-FOXO semble jouer un rôle prépondérant dans la longévité, et ce de façon conservée au cours de l'évolution, du ver jusqu'à l'homme. De nombreuses questions restent en suspens: quels sont les rôles de cette voie de longévité dans les pathologies liées à l'âge ? Quelle est l'importance de la voie insuline-FOXO dans les bénéfices liés à la restriction calorique? Comment moduler efficacement cette voie par une approche pharmacologique? Dans quels cellules et tissus cette voie exerce-t-elle le plus d'influence sur la durée de vie ? Existe-t-il un compromis entre la longévité et d'autres phénotypes, comme la fertilité ? Les réponses à ces questions auront une influence déterminante pour imaginer une stratégie pour «bien vieillir». $\diamond$

\section{SUMMARY}

Aging and the control of the insulin-FOXO signaling pathway

Aging is a complex process that is accompanied by the onset of a series of age-related diseases, including Alzheimer's disease. Aging is controlled by a combination of genetic and environmental factors. Among the genes that regulate aging, the insulin-FOXO signaling pathway plays a central role, as this pathway regulates lifespan in multiple species, such as worms, flies, and mice. In humans, exceptional longevity - being a centenarian - is also associated with genetic variation in this insulin-FOXO pathway. Recent evidence indicates that the FOXO family of transcription factors plays a key role in the self-renewal of adult and embryonic stem cells, which could contribute to tissue regeneration. Understanding the mechanisms underlying aging should help better prevent and treat age-dependent diseases. $\diamond$

\section{CONFLITS D'INTÉRÊTS}

L'auteur déclare n'avoir aucun conflit d'intérêts concernant les données publiées dans cet article.

\section{RÉFÉRENCES}

1. Kenyon C, Chang J, Gensch $\varepsilon$, et al. A C. elegans mutant that lives twice as long as wild type. Nature 1993 ; $366: 461-4$.

2. Kenyon CJ. The genetics of ageing. Nature $2010 ; 464: 504-12$.

3. Tatar M, Kopelman A, Epstein D, et al. A mutant Drosophila insulin receptor homolog that extends life-span and impairs neuroendocrine function. Science $2001 ; 292$ : 107-10.

4. Clancy DJ, Gems D, Harshman LG, et al. Extension of life-span by loss of CHICO, a Drosophila insulin receptor substrate protein. Science $2001 ; 292$ : 104-6.

5. Holzenberger M, Dupont J, Ducos B, et al. IGF-1 receptor regulates lifespan and resistance to oxidative stress in mice. Nature $2003 ; 421: 182-7$.

6. Bluher M, Kahn BB, Kahn CR. Extended longevity in mice lacking the insulin receptor in adipose tissue. Science $2003 ; 299: 572-4$

7. Cohen $\varepsilon$, Paulsson JF, Blinder P, et al. Reduced IGF-1 signaling delays ageassociated proteotoxicity in mice. Cell 2009 ; 139 : 1157-69.

8. Suh Y, Atzmon G, Cho MO, et al. Functionally significant insulin-like growth factor I receptor mutations in centenarians. Proc Natl Acad Sci USA 2008 ; $105: 3438-42$.

9. Greer \&L, Brunet A. FOXO transcription factors at the interface between longevity and tumor suppression. Oncogene $2005 ; 24: 7410-25$

10. Brunet A, Park J, Tran H, et al. Protein kinase SGK mediates survival signals by phosphorylating the forkhead transcription factor FKHRLI (FOXO3a). Mol Cell Biol $2001 ; 21: 952-65$.

11. Kops GJ, de Ruiter ND, De Vries-Smits AM, et al. Direct control of the Forkhead transcription factor AFX by protein kinase B. Nature $1999 ; 398: 630-4$.

12. Biggs WH, 3rd, Meisenhelder J, Hunter T, et al. Protein kinase B/Aktmediated phosphorylation promotes nuclear exclusion of the winged helix transcription factor FKHR1. Proc Natl Acad Sci USA 1999 ; 96 : 7421-6.

13. Brunet A, Bonni A, Zigmond MJ, et al. Akt promotes cell survival by phosphorylating and inhibiting a Forkhead transcription factor. Cell 1999 ; 96: 857-68.

14. Tran $H$, Brunet A, Grenier JM, et al. DNA repair pathway stimulated by the forkhead transcription factor FOX03a through the Gadd45 protein. Science $2002 ; 296: 530-4$

15. Kops GJ, Dansen TB, Polderman PE, et al. Forkhead transcription factor FOXO3a protects quiescent cells from oxidative stress. Nature $2002 ; 419$ : 316-21. 


\section{RÉFÉRENCES}

16. Medema RH, Kops GJ, Bos JL, Burgering BM. AFX-like Forkhead transcription factors mediate cellcycle regulation by Ras and PKB through p27kipl. Nature $2000 ; 404: 782-7$.

17. Nemoto $S$, Finkel T. Redox regulation of forkhead proteins through a p66shc-dependent signaling pathway. Science $2002 ; 295: 2450-2$.

18. Murphy CT, McCarroll SA, Bargmann Cl, et al. Genes that act downstream of DAF- 16 to influence the lifespan of Caenorhabditis elegans. Nature $2003 ; 424: 277-83$.

19. Calnan DR, Brunet A. The FoxO code. Oncogene $2008 ; 27: 2276-88$.

20. Greer EL, Oskoui PR, Banko MR, et al. The energy sensor AMP-activated protein kinase directly regulates the mammalian FOX03 transcription factor. J Biol Chem 2007 ; 282 : 30107-19.

21. Greer $\varepsilon L$, Dowlatshahi D, Banko MR, et al. An AMPK-FOXO pathway mediates longevity induced by a novel method of dietary restriction in C. elegans. Curr Biol 2007 ; 17 : 1646-56.

22. Brunet A, Sweeney LB, Sturgill JF, et al. Stress-dependent regulation of FOXO transcription factors by the SIRTl deacetylase. Science 2004 ; 303 : 2011-5.

23. Motta MC, Divecha N, Lemieux M, et al. Mammalian SIRTl represses forkhead transcription factors. Cell $2004 ; 116: 551-63$.

24. Aoki M, Jiang H, Vogt PK. Proteasomal degradation of the Fox01 transcriptional regulator in cells transformed by the P3k and Akt oncoproteins. Proc Natl Acad Sci USA 2004 ; 101 : 13613-7.

25. Renault VM, Thekkat PU, Hoang KL, et al. The pro-longevity gene Fox 03 is a direct target of the p53 tumor suppressor. Oncogene $2011 ; 30$ : 3207-21.

26. Hwangbo DS, Gershman B, Tu MP, et al. Drosophila dFOXO controls lifespan and regulates insulin signalling in brain and fat body. Nature $2004 ; 429$ : 562-6.

27. Giannakou ME, Goss M, Junger MA, et al. Long-lived Drosophila with overexpressed dFOXO in adult fat body. Science $2004 ; 305: 361$.

28. Henderson ST, Johnson TE. daf- 16 integrates developmental and environmental inputs to mediate aging in the nematode Caenorhabditis elegans. Curr Biol $2001 ; 11: 1975-80$.

29. Willcox BJ, Donlon TA, He $Q$, et al. FOX03A genotype is strongly associated with human longevity. Proc Natl Acad Sci USA 2008 ; 105 : 13987-92.

30. Pawlikowska L, Hu D, Huntsman S, et al. Association of common genetic variation in the insulin/ IGFl signaling pathway with human longevity. Aging Cell $2009 ; 8: 460-72$.
31. Flachsbart F, Caliebe A, Kleindorp R, et al. Association of FOXO3A variation with human longevity confirmed in German centenarians. Proc Natl Acad Sci USA $2009 ; 106: 2700-5$.

32. Anselmi CV, Malovini A, Roncarati R, et al. Association of the FOX03A locus with extreme longevity in a southern Italian centenarian study. Rejuvenation Res $2009 ; 12: 95-104$.

33. Li Y, Wang WJ, Cao H, et al. Genetic association of FOXO1A and FOXO3A with longevity trait in Han Chinese populations. Hum Mol Genet 2009 ; 18 : 4897-904.

34. Renault VM, Rafalski VA, Morgan AA, et al. Fox03 regulates neural stem cell homeostasis. Cell Stem Cell $2009 ; 5: 527-39$.

35. Tothova Z, Gilliland DG. Fox0 transcription factors and stem cell homeostasis: insights from the hematopoietic system. Cell Stem Cell 2007 ; $1: 140-52$.

36. Paik JH, Ding Z, Narurkar R, et al. FoxOs cooperatively regulate diverse pathways governing neural stem cell homeostasis. Cell Stem Cell $2009 ; 5$ : 540-53.

37. Miyamoto K, Araki Ky, Naka K, et al. Foxo3a is essential for maintenance of the hematopoietic stem cell pool. Cell Stem Cell $2007 ; 1: 101-12$.

38. Zhang X, Yalcin S, Lee DF, et al. FOXOl is an essential regulator of pluripotency in human embryonic stem cells. Nat Cell Biol $2011 ; 13$ : 1092-9.

39. Brunet $A$. Les multiples actions des facteurs de transcription Foxo. Med Sci (Paris) $2004 ; 20: 856-9$.

40. Holzenberger $M$. Les récepteurs centraux de l'IGF-l contôlent la longévité chez la souris. Med Sci (Paris) $2009 ; 25: 371-6$.

41. Tothova Z, Mercher T. Fox0 : stress vie éternelle. Med Sci (Paris) 2007 ; 23 : 466-7.

42. Rimmelé $P$, Zhang $Y$, Ghaffari $S$. Rôle des facteurs de transcription Fox 0 dans la maintenance des cellules souches. Med Sci (Paris) $2012 ; 28: 250-4$.

\section{TIRÉS À PART}

A. Brunet

\section{Collection SCIENCE ET BIOMÉDECINE}

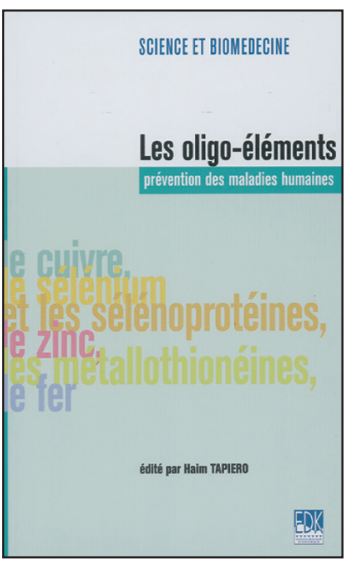

ISBN : 2-84254-107-3 64 pages

$10 €+3 €$ de port $=13 €$ TTC

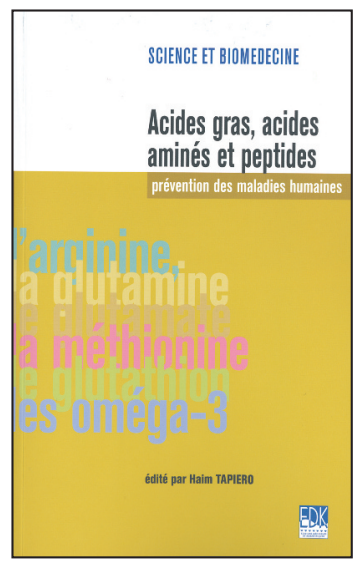

ISBN : 2-84254-108-1 80 pages $12 €+3 €$ de port $=\mathbf{1 5} €$ TTC

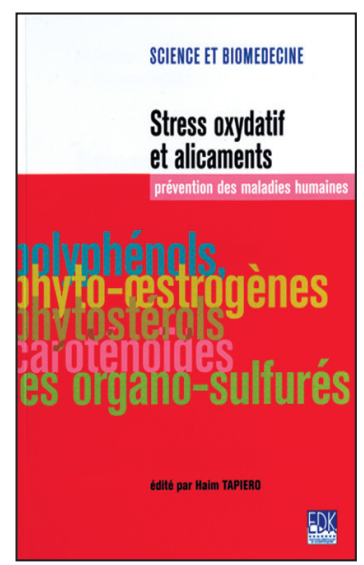

ISBN : 2-84254-111-1 86 pages $14 €+3 €$ de port $=17 €$ TTC

\section{Bon de commande}

À retourner à EDK, 25, rue Daviel - 75013 Paris

Tél. : 0158101905 - Fax : 0143293262 - E-mail : edk@edk.fr

NOM :

Prénom :

Adresse :

Code postal :

Ville :

Pays :

Fonction :

Je souhaite recevoir soit un total de

$\square$ Par chèque, à l'ordre de $\mathbf{E} \mathbf{D}$ K

$\square$ Par carte bancaire : $\square$ Visa $\square$ Eurocard/Mastercard

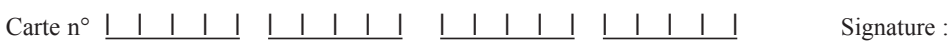

Date d'expiration: $\quad \leq 1|1|$

$N^{\circ}$ de contrôle au dos de la carte : $\quad$ । $\quad$ | $\quad$ | 